\title{
Steady-State Analysis of Switching Converters via Frequency-Domain Circuit Equivalents
}

\author{
Riccardo Trinchero, Student Member, IEEE, Paolo Manfredi, Member, IEEE, Igor S. Stievano, Senior \\ Member, IEEE, Flavio G. Canavero, Fellow, IEEE
}

\begin{abstract}
This brief presents a frequency-domain approach for the steady-state analysis of pulse-width modulated converters and switched circuits with non-ideal switching behavior. The proposed strategy generalizes recent methodologies based on the Fourier expansion of the steady-state responses of a periodically switching circuit and on the simulation of an augmented linear time-invariant system. This system is now also given an interpretation in terms of an equivalent circuit, which is simulated at a single frequency point to solve for all the harmonics. The method offers a modular topological approach that is combined with standard tools for circuit analysis and enables the simulation of networks with an arbitrary number of switches and driving mechanisms. Single, multiple, and possibly non-ideal commutation events within the switching period are handled in the same framework, without additional complexity. The technique allows for the full frequency-domain characterization of both the functional and the noisy behavior of the circuit responses. The feasibility and strength are demonstrated via comparisons with simulations and measurements on two application examples, i.e. a full-bridge single-phase inverter and a dc-dc boost converter.
\end{abstract}

Index Terms-Circuit simulation, harmonic analysis, periodically switched linear circuits, pulse width modulation inverters, SPICE, switching converters.

\section{INTRODUCTION}

Periodically switched linear (PSL) circuits represent a wide and important class of time-varying electrical networks that are used to describe the behavior of a number of modern devices. A relevant example is provided by switching power converters, which are massively used to supply energy to electrical and electronic equipment and appliances [1]. PSL circuits exhibit a complex dynamical behavior arising from the periodic activity of the switches and require suitable methods to accurately model and predict, in the design phase, the voltage and current waveforms at their steady state. In particular, the currents absorbed by switching circuits are the main sources of conducted emissions (CEs), which need to be fully characterized to comply with the current electromagnetic compatibility (EMC) regulations specified in terms of maximum levels over a wideband frequency spectrum [2].

This work was partially supported by the Research Foundation Flanders (FWO-Vlaanderen)

R. Trinchero, I. S. Stievano and F. G. Canavero are with the EMC Group, Department of Electronics and Telecommunications, Politecnico di Torino, 10129 Torino, Italy (e-mail: riccardo.trinchero@polito.it, igor.stievano@polito.it, flavio.canavero@polito.it).

P. Manfredi is with the Electromagnetics Group, Department of Information Technology, Ghent University, 9000 Gent, Belgium (e-mail: paolo.manfredi@ugent.be).

Copyright (c) 2015 IEEE. Personal use of this material is permitted. However, permission to use this material for any other purposes must be obtained from the IEEE by sending an email to pubs-permissions@ieee.org.
Some modeling approaches provide a simplified, averaged behavior of the steady-state response [3]. These methods, however, generate low-frequency models that cannot reproduce the wide-band behavior of switching circuits. Alternative simulation techniques are available that provide the frequencydomain representation of the steady-state response [4]-[9]. Operating directly in the frequency domain has the relevant advantage of avoiding the calculation of possibly long transients and any issue related to the proper choice of integration time step and waveform windowing, which may impact the accuracy and the efficiency of the simulation. Nonetheless, the complex mathematical formulation and the cumbersome technical solutions burden the analysis of linear switching circuits with arbitrary network configurations and driving mechanisms.

To overcome the aforementioned limitations, a more straightforward framework has been recently proposed for the class of PSL circuits [10], [11], which are interpreted in terms of augmented linear time-invariant (LTI) networks whose frequency-domain solution directly provides the harmonics of the steady-state responses. Such a LTI network is readily built from the topological structure of the original switching circuit. Unfortunately, the technique is limited to ideal switches with single and instantaneous transitions within the period and cannot be applied, as is, to a wide class of more practical circuits that involve multiple commutations and/or non-ideal (e.g., noisy) switching dynamics.

Therefore, the aim of this brief is to extend the existing methodology by introducing an improved modeling framework that can deal with an arbitrary behavior of the switches, including the relevant cases of pulse-width modulation (PWM) and of real-life MOS and diode elements in switched-mode power converters. Furthermore, an equivalent circuit interpretation of the pertinent augmented LTI network is also provided, thus additionally rendering the approach compatible with advanced circuit solvers such as SPICE. The equivalent models are automatically generated via a modular and topological approach. A general solution method is thus established that overcomes the inherent limitations of an ad-hoc derivation and solution of generic switching circuits (e.g., via statespace circuit descriptions). The above feature also enables the analysis of practical circuits with a sufficiently large number of switches and different modulation schemes.

\section{Proposed Simulation Framework}

This section summarizes the proposed simulation framework for the solution of a generic PSL circuit like the one 
shown in Fig. 1, which is a representative example with the key elements of this class of circuits (i.e. resistors, dynamical elements, and switches) and allows for a comprehensive illustration of the method. The switch $S$ is a time-varying element characterized by a periodic behavior with either a single commutation (SC) or a multiple commutation (MC) within the period $T$. The switch behavior is represented, for the moment, by logical binary signals, with the levels "one" and "zero" corresponding to the closed and open switch, respectively. The unified handling of both SC and MC operation modes within the same simulation framework allows for the application of the method to a wide class of switching circuits, ranging from dc-dc converters to PWM dc-ac inverters, thus extending the state-of-the-art technique available in the literature. Later on, the switching behavior is further generalized to arbitrary dynamics.
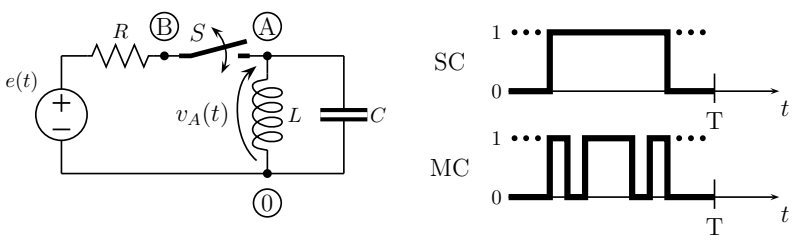

Figure 1. Example of a dynamical PSL circuit. The switch is driven by single or multiple commutation events within the switching period $T$, referred to as SC and MC operation modes, respectively.

\section{A. Frequency-Domain Representation and Constitutive Rela- tions}

Analogously to harmonic balance simulations, for the frequency-domain analysis of a PSL circuit the independent voltage source is defined as a cisoidal excitation $e(t)=$ $\left(E_{0} / 2 \pi\right) \exp \left(j \omega_{0} t\right)$ with angular frequency $\omega_{0}$, and the periodic response at the steady state is expressed as a truncated sum of delta functions. E.g., for the voltage $v_{A}(t)$ in Fig. 1:

$$
V_{A}(\omega) \approx \sum_{n=-N}^{+N} V_{A, n} \delta\left(\omega-n \omega_{c}-\omega_{0}\right),
$$

where $V_{A, n}$ are the $2 N+1$ Fourier coefficients (harmonics) of the voltage $v_{A}(t)$, and $\omega_{c}=2 \pi / T$. Analogous approximations hold for all the other voltages and currents. Based on the above interpretation of the circuit variables, appropriate constitutive relations are derived for the voltage and current harmonics of both the LTI elements and the switches.

The substitution of (1) into the $i$ - $v$ constitutive relations of the standard LTI elements such as resistors, capacitors and inductors, yields the augmented relations between the voltage and current harmonics collected in the first three rows of Table I [11]. It is worth noting that in this case no coupling between the harmonics is involved, in agreement with the wellknown concepts underlying the phasor analysis of linear and time-invariant circuits. The frequency-domain current-voltage characteristic of a generic switching element is given in the last row of the table instead. Contrary to classical LTI circuits, the presence of a PSL element couples the voltage and current harmonics, which can no longer be computed independently.
Table I

RELATIONSHIPS BETWEEN THE VOLTAGE AND CURRENT HARMONICS FOR THE MAIN LTI ELEMENTS AND A SWITCH $(n, k=-N, \ldots,+N)$.

\begin{tabular}{|c|c|c|}
\hline element & constitutive relation & harmonic relation \\
\hline$\rightarrow \underbrace{i}_{v}$ & $v=R i$ & $V_{n}=R I_{n}$ \\
\hline 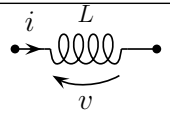 & $v=L \frac{d i}{d t}$ & $V_{n}=j n \omega_{c} L I_{n}$ \\
\hline$\left.\leftrightarrow\right|_{\frac{1}{v}} ^{i}$ & $i=C \frac{d v}{d t}$ & $I_{n}=j n \omega_{c} C V_{n}$ \\
\hline$\rightarrow \frac{i s}{\frac{S}{v}}$ & 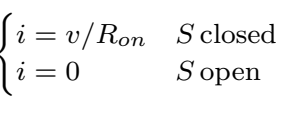 & $\begin{array}{l}I_{n}=\sum_{m, k} Y_{m} V_{k} \\
\text { for } m+k=n\end{array}$ \\
\hline
\end{tabular}

The coefficients $Y_{m}$ are complex numbers computed as the Fourier transform of the time-domain periodic behavior of the switch admittance, i.e.

$$
Y_{m}=\frac{1}{T} \int_{0}^{T} \frac{1}{R_{o n}} \Pi(t) \exp \left(-j m \omega_{c} t\right) d t
$$

$m=-2 N, \ldots, 0, \ldots, 2 N$, where $R_{o n}$ is the series resistance of the switch, whilst $\Pi(t)$ is the function defining the switch operation as shown in Fig. 1.

\section{B. Modeling of the SC and MC Switching Modes}

The Fourier transform (2) is computed according to the behavior of the function $\Pi(t)$. If the switch $S$ operates in $\mathrm{SC}$ mode, it is closed once during the period $T$, thus leading to the following periodic behavior:

$$
\Pi(t)=\Pi_{S C}(t)= \begin{cases}1 & \text { for } t \in\left[t^{*}, t^{*}+D T\right] \\ 0 & \text { otherwise }\end{cases}
$$

For this simple case, the coefficients $Y_{m}$ are computed analytically as [11]

$$
Y_{S C, m}=\frac{1}{R_{o n} T} \frac{\exp \left(-j m \omega_{c} t^{*}\right)-\exp \left(-j m \omega_{c}\left(t^{*}+D T\right)\right)}{j m \omega_{c}} .
$$

The previous scenario is now generalized to handle the more complex MC switching behavior. In principle, this can be interpreted as a sum of SC square wave functions, i.e., $\Pi_{M C}(t)=\sum_{i=1}^{M} \Pi_{S C, i}(t)$, with $M$ the number of commutations occurring in a single period. Given the linearity of the Fourier operator, the coefficients $Y_{M C, m}$ are in turn computed as a superposition of the analytical result (4).

Nonetheless, an alternative and more practical approach is to numerically compute these entries by replacing the continuous-time Fourier transform (2) with a discrete Fourier transform (DFT) via the following relation:

$$
Y_{M C, m}= \begin{cases}\frac{1}{R_{o n} N_{s}} \sum_{k=0}^{N_{s}-1} \Pi[k] \exp \left(-\frac{j k 2 \pi m}{N_{s}}\right) & \text { if } m \geq 0 \\ \frac{1}{R_{o n} N_{s}} \sum_{k=0}^{N_{s}-1} \Pi[k] \exp \left(-\frac{j k 2 \pi\left(N_{s}+m\right)}{N_{s}}\right) & \text { if } m<0\end{cases}
$$


where $N_{s}=4 N+1$ and $\Pi[k]=\Pi\left(k \Delta_{t}\right)$ is the sampled version of the continuous signal $\Pi(t)$ on a single period $T$ at equispaced intervals $\Delta_{t}=T / N_{s}$.

The number of samples $N_{s}$ has to be greater than $4 N+1$ to obtain all the entries in Table I (from $Y_{-2 N}$ to $Y_{2 N}$ ). It is worth to remark that, since the spectrum of a generic switching signal $\Pi(t)$ is of infinite bandwidth, the sampling will inevitably lead to aliasing. In order to reduce the aliasing effect on the first $2 N+1$ harmonics of the spectrum of the discrete signal $\Pi[k]$, a number of samples $N_{s}>8 N$ is suggested.

As a further generalization, the function $\Pi(t)$ can be replaced by any normalized periodic function that describes the possible non-ideal behavior of the switch. The typical example is provided by a component that switches between the "on" and the "off" state with a finite transition time and arbitrary (e.g., noisy) commutation. This alternative interpretation is particularly useful when the information on the actual switching behavior of real components is available (e.g., from measurements) and can be used to improve the predicted results, as will be shown in Section IV.

\section{Equivalent Circuit Interpretation}

Assuming that the node voltages and the branch currents are expanded according to (1), a corresponding augmented network is created by first associating a node to each voltage harmonic coefficient. The resulting network has thus a total number of nodes that is $2 N+1$ times larger. These nodes are then connected with suitable elements as described in the following, in accordance with the original circuit topology.

For the case of a resistor $R$, the same current-voltage relationship is preserved for all the harmonics (first row of Table I), and the resistor is simply replicated between the pertinent nodes. For each harmonic of a capacitor instead, the characteristic equation in the second row of Table I is mapped into a modified capacitance $\tilde{C}_{n}$, computed via the following admittance equation:

$$
j\left(\omega_{0}+n \omega_{c}\right) C=j \Omega \tilde{C}_{n},
$$

where $\Omega>0$ is the simulation frequency. The modified capacitances are thus defined as $\tilde{C}_{n}=\frac{\omega_{0}+n \omega_{c}}{\Omega} C$. It should be noted that the choice of the simulation frequency $\Omega$ is arbitrary and the modified capacitance is computed accordingly. An inductor is similarly mapped into modified inductances.

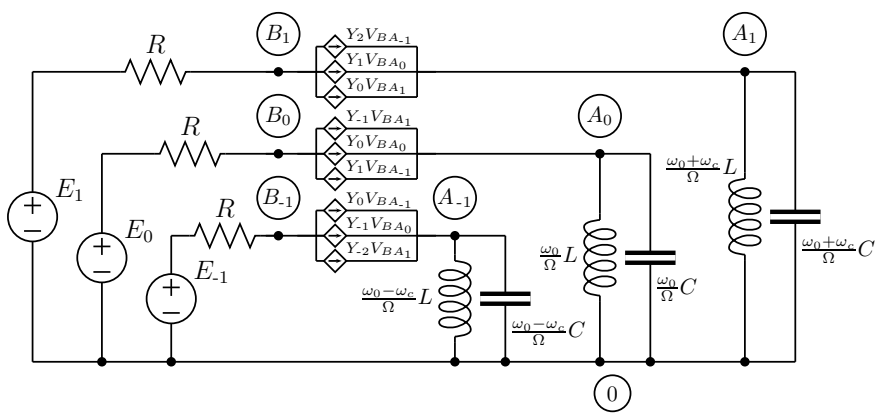

Figure 2. Augmented equivalent LTI model of the switching circuit of Fig. 1 for $N=1$. The notation $V_{B A_{n}}=V_{B_{n}}-V_{A_{n}}$ is used.
So far, the circuit interpretation for LTI elements preserves the component type, as there is no coupling between their voltage and current harmonics. On the other hand, the coupled equation of a PSL element is implemented by introducing, for each term $Y_{m}=G_{m}+j B_{m}$, a dependent current source that exhibit an admittance transfer function in the form of $k_{0, m}+j \Omega k_{1, m}$, with $k_{0, m}=G_{m}$ and $k_{1, m}=B_{m} / \Omega$. Hence, each PSL component is replaced by dependent sources with a transadmittance equal to the Fourier coefficients of the switch time-domain behavior. Finally, independent sources are also expressed via (1), and the corresponding Fourier coefficients are suitably connected to the nodes of the augmented network.

For the sake of illustration, Fig. 2 shows the augmented LTI counterpart of the example circuit of Fig. 1. To limit the circuit size, only three harmonics, corresponding to $n=-1,0,+1$, are considered. The different models for each element are connected based on the same topological structure as the original network. The sources $E_{-1}, E_{0}$ and $E_{1}$ are the Fourier coeffients of the time-domain source $e(t)$. For the frequent case of dc sources $\left(\omega_{0}=0\right)$, only the generator $E_{0}$ is non-null. The resulting network is a coupled LTI circuit that is solved once at a single simulation frequency $\Omega$ to simultaneously retrieve all the $2 N+1$ harmonics describing the steady-state voltage and current responses of the original PSL circuit and resulting from the excitation at the angular frequency $\omega_{0}$. The equivalent circuit is compatible with advanced commercial SPICE-type simulators and is easily implemented via available components. Alternatively, the modified nodal analysis (MNA) representation [12] of the network is readily generated from circuit inspection and it is solved via a single linear inversion, e.g., in MATLAB.

The illustration of Fig. 2 contains all the key building blocks that allow to produce the equivalent models of more complex circuits as those considered in the following application examples. Furthermore, the outlined topological procedure allows for the automatic and transparent generation of the augmented LTI equivalent starting from the original netlist of any arbitrary PSL circuit. It is relevant to point out that no specific assumption has been made on the switch operation, and the equivalent model applies to arbitrary commuting behaviors.

\section{Application \# 1: Numerical Simulation}

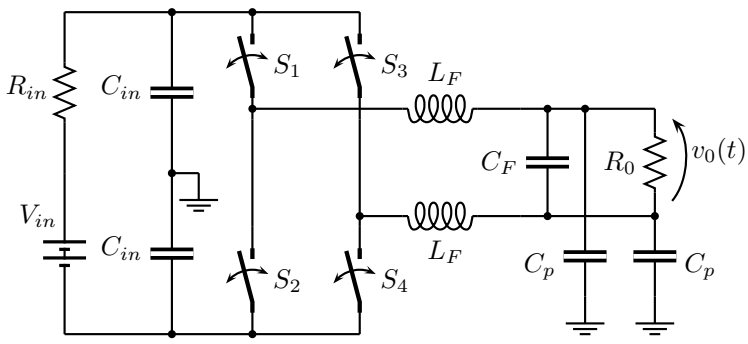

Figure 3. Full-bridge single-phase inverter. The circuit elements take the following values: $V_{i n}=15 \mathrm{~V}, R_{i n}=1 \Omega, C_{i n}=47 \mu \mathrm{F}, L_{F}=2 \mathrm{mH}$, $C_{F}=30 \mu \mathrm{F}, C_{p}=300 \mathrm{pF}$ and $R_{0}=20 \Omega$.

The effectiveness of the proposed method is demonstrated on the PWM full-bridge single-phase inverter [1] shown in 
Fig. 3. The switches $S_{k}, k=1, \ldots, 4$ are driven by four PWM signals obtained by comparing a reference sinusoidal signal $r(t)$ of frequency $f_{r}=60 \mathrm{~Hz}$ with a companion periodic triangular carrier $c(t)$ of frequency $f_{c}=960 \mathrm{~Hz}$. The behavior of the switches $S_{1}$ and $S_{3}$ of the H-bridge is defined by means of the normalized functions $\Pi_{1}(t)$ and $\Pi_{3}(t)$ shown in Fig. 4. The remaining switches $S_{2}$ and $S_{4}$ behave according to the functions $\Pi_{2}(t)=1-\Pi_{1}(t)$ and $\Pi_{4}(t)=1-\Pi_{3}(t)$, so that a short circuit across the voltage source $V_{i n}$ is avoided.

Switch control signals

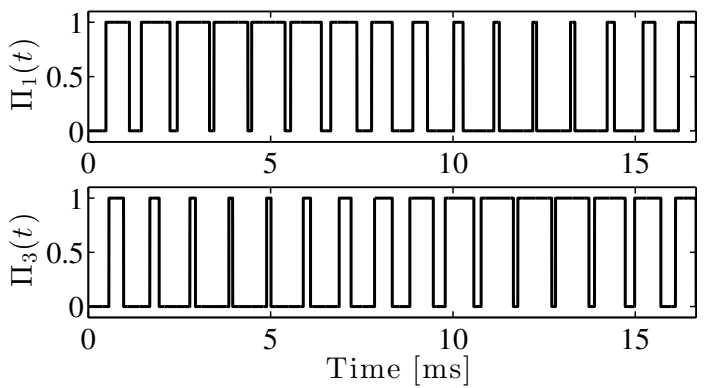

Figure 4. Operation of the switches $S_{1}$ and $S_{3}$ in the scheme of Fig. 3.

The methodology introduced in Section II allows to obtain a LTI equivalent of the converter, where the time-varying PWM switches are replaced by their augmented frequencydomain representations. This equivalent representation of the converter has been implemented both in a MATLAB script, by means of the MNA formalism, and in SPICE, by considering the circuital interpretation discussed in Section II-C. Fig. 5 shows the spectrum (top panel) and the corresponding steadystate time-domain response (bottom panel) of the output voltage $v_{0}$, predicted with the proposed frequency-domain approach in MATLAB (red line) and SPICE (inner black line). For comparison, the reference result calculated with a transient Simulink simulation with $t_{\text {step }}=0.1 \mu \mathrm{s}$ is also provided (outer gray line). The curves in the figure highlight the excellent accuracy of the proposed solution in reproducing the steadystate response without the initial transient. To account for the high-frequency components of the steady-state response, the expansion order is set to a sufficiently large value of $N=180$. In MATLAB, the proposed method requires a computational time of 1.04 s only, and achieves a speed-up of $30 \times$ with respect to the transient simulation.

\section{APPLICATION \#2: EXPERIMENTAL VERIFICATION}

The proposed methodology is now used to reproduce the measured spectrum of the differential mode (DM) CE of the boost converter of Fig. 6. According to the EMC standards, the $\mathrm{CE}$ of the converter is obtained by measuring the voltages $v_{L G}$ and $v_{N G}$ at the output ports of a LISN (Line Impedance Stabilization Network) and then the DM emissions are computed offline as $V_{D M}=\left(V_{L G}-V_{N G}\right) / 2$.

The boost converter operating at the steady state is considered as a PSL circuit, with the MOS and the diode suitably replaced by two periodic switches of frequency $f_{c}$. Specifically, two different switching behaviors are considered, as shown in
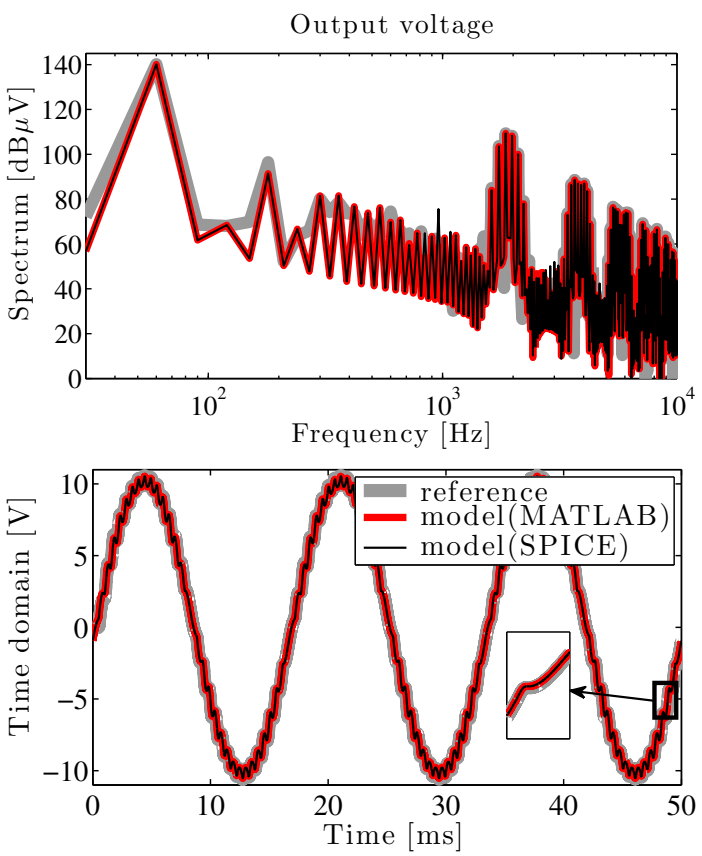

Figure 5. Steady-state behavior of the inverter output voltage $v_{0}$ in both frequency (top panel) and time (bottom panel) domain. Gray line: reference transient simulation; red and black lines: predictions of the proposed technique implemented in MATLAB and SPICE, respectively.

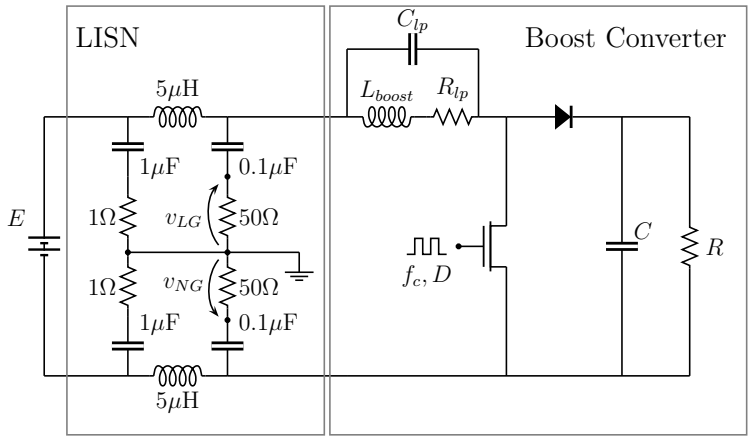

Figure 6. Experimental setup for the measurement of the DM CE of a boost converter. The converter operates in continuous mode with switching frequency $f_{c}=50 \mathrm{kHz}$, duty cycle $D=50 \%$ and constant input voltage $E=5 \mathrm{~V}$. The component values are as follows: $L_{\text {boost }}=470 \mu \mathrm{H}, R_{l p}=$ $1.3 \Omega, C_{l p}=27 p F, C=470 \mu \mathrm{F}$ and $R=150 \Omega$.

Fig. 7: i) an ideal and complementary commutation (dashed lines), in which the MOS is off when the diode is on, and vice versa, and ii) the actual measured behavior of the voltages across the diode and the MOS elements (solid lines). In both cases, the corresponding augmented network is generated from the schematic of Fig. 6 using the rules provided in Section II and it is simulated in the frequency domain, leading to the prediction of the $\mathrm{CE}$ spectrum. The number of harmonics is set to $N=f_{\max } / f_{c}=600$ to cover the entire frequency band specified by EMC standards $\left(f_{\max }=30 \mathrm{MHz}\right)$.

Fig. 8 compares the measured (solid gray line) and predicted (black and red lines) CEs for the two different operations of the switches. It is worth noting that the result obtained by assuming an ideal behavior of the switching elements (black line) fails to predict the spectral harmonics placed at the even multiples of the switching frequency $f_{c}$. However, when the 


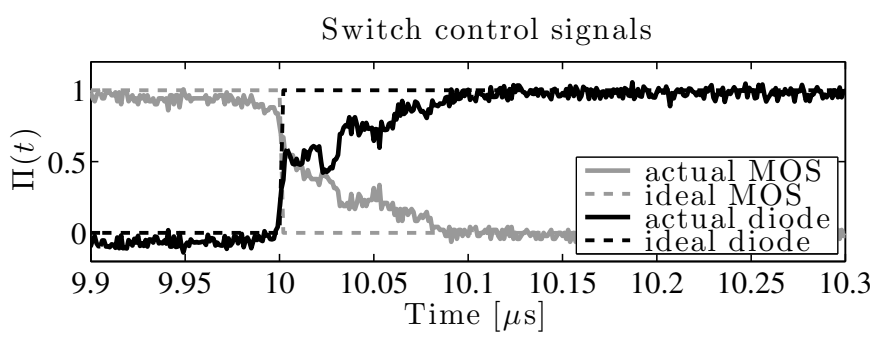

Figure 7. Normalized measured voltages across the diode and the MOS components.

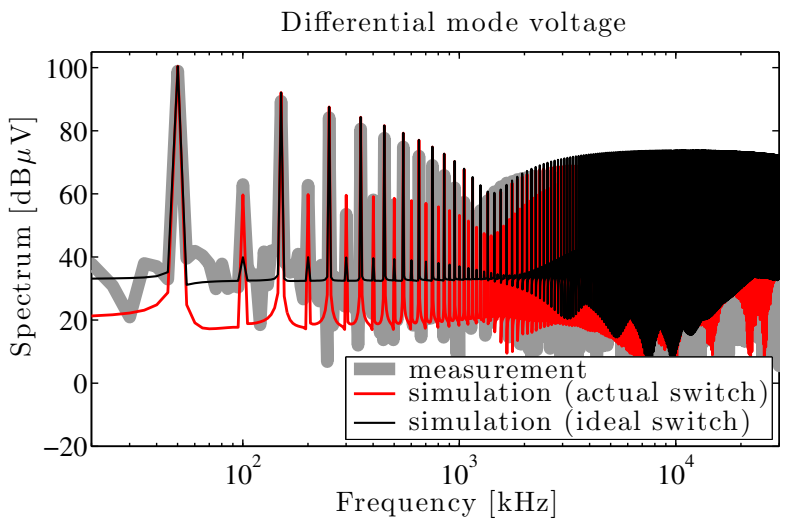

Figure 8. Spectrum of the boost DM CE. The measured behavior (gray line) is compared against the prediction of the proposed frequency-domain method with both the ideal (black line) and the measured (red line) switch operation.

actual behavior of the switches is used in the simulation, a far better accuracy is indeed achieved (see the red line), with improvements in the prediction of the even harmonics and of the high-frequency response. Such a correction is not straightforward in a traditional time-domain simulation and could not have been implemented in the framework in [10], [11].

To further illustrate the inherent, yet critical issues in the transient simulation of switched networks, Fig. 9 shows a time-domain simulation of the differential voltage $v_{D M}(t)$ in SPICE. A long transient is required for the voltage to reach the steady state, which is readily and accurately captured with the proposed frequency-domain approach instead (see the insert). This makes the presented method $18 \times$ faster $(7.5 \mathrm{~s}$ vs $135.8 \mathrm{~s})$ despite the augmented size of the equivalent LTI network.

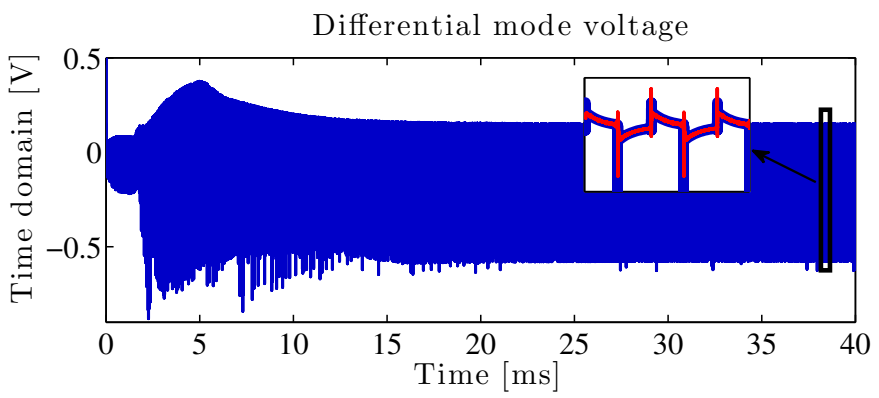

Figure 9. Time-domain response of the boost DM voltage. The reference SPICE simulation (blue line) exhibits a long transient. The behavior at the steady state is well predicted by the proposed method (red curve in the insert).

\section{CONCLUSIONS}

In this brief, a frequency-domain approach for the steadystate simulation of switching converters is proposed. The method applies to a broad class of linear dynamical circuits consisting of classical LTI components and switches driven by PWM signals and/or with possibly non-ideal commutation events. A flexible and comprehensive framework is derived, where the original time-varying network is interpreted in terms of an equivalent augmented LTI circuit. The new network variables correspond to the harmonics of the steady-state circuit responses and are calculated via a single circuit simulation. The equivalent circuit is compatible with standard SPICEtype solvers. The method has been applied to the prediction of the steady-state behavior of a PWM inverter and a dc$\mathrm{dc}$ boost converter with measured behavior of its switching components, and excellent agreement and simulation speedup were observed with respect to the reference results. Other possible and envisaged applications include the robust design and optimization of this class of circuits.

\section{REFERENCES}

[1] M. H. Rashid, Power Electronics: Circuits, Devices and Applications. 3rd edn., Prentice Hall, 2004.

[2] E. Rondon-Pinilla, F. Morel, C. Vollaire, and J.-L. Schanen, "Modeling of a buck converter with a SiC JFET to predict EMC conducted emissions," IEEE Trans. Power Electron., vol. 29, no. 5, pp. 2246-2260, May 2014.

[3] A. Davoudi, J. Jatskevich, and T. De Rybel, "Numerical state-space average-value modeling of PWM DC-DC converters operating in DCM and CCM," IEEE Trans. Power Electron., vol. 21, no. 4, pp. 1003-1012, Jul. 2006.

[4] M.-L. Liou, "Exact analysis of linear circuits containing periodically operated switches with applications," IEEE Trans. Circuit Theory, vol. 19, no. 2, pp. 146-154, Mar. 1972.

[5] S. R. Sanders, J. M. Noworolski, X. Z. Liu, and G. C. Verghese, "Generalized averaging method for power conversion circuits," IEEE Trans. Power Electron., vol. 6, no. 2, pp. 251-259, Apr. 1991.

[6] F. Yuan and A. Opal, "Noise and sensitivity analysis of periodically switched linear circuits in frequency domain," IEEE Trans. Circuits Syst. I, Reg. Papers, vol. 47, no. 7, pp. 986-998, Jul. 2000.

[7] F. Wang, H. Zhang, and X. Ma, "Analysis of slow-scale instability in boost PFC converter using the method of harmonic balance and floquet theory," IEEE Trans. Circuits Syst. I, Reg. Papers, vol. 57, no. 2, pp. 405414, Feb. 2010.

[8] J. Liang and W.-H. Liao, "Steady-state simulation and optimization of class-E power amplifiers with extended impedance method," IEEE Trans. Circuits Syst. I, Reg. Papers, vol. 58, no. 6, pp. 1433-1445, Jun. 2011.

[9] H. Behjati, L. Niu, A. Davoudi, and P. L. Chapman, "Alternative timeinvariant multi-frequency modeling of PWM DC-DC converters," IEEE Trans. Circuits Syst. I, Reg. Papers, vol. 60, no. 11, pp. 3069-3079, Nov. 2013.

[10] R. Trinchero, I. S. Stievano, and F. G. Canavero, "Steady-state response of periodically switched linear circuits via augmented time-invariant nodal analysis," J. Elect. Comput. Eng., vol. 2014, article ID 198273, 2014.

[11] R. Trinchero, I. S. Stievano, and F. G. Canavero, "Steady-state analysis of switching power converters via augmented time-invariant equivalents", IEEE Trans. Power Electron., vol. 29, no. 11, pp. 5657-5661, Nov. 2014.

[12] C.-W. Ho, A. Ruehli, and P. Brennan, "The modified nodal approach to network analysis," IEEE Trans. Circuits Syst., vol. 22, no. 6, pp. 504509, Jun. 1975. 